\title{
La ecología de las instituciones educativas: entorno, control social y comportamiento en colegios de Colombia
}

Recibido: 08 de junio de 2020 - Aceptado: 16 de noviembre de 2020

Doi: https://doi.org/10.12804/revistas.urosario.edu.co/economia/a.10023

\author{
Ximena Dueñas Herrera* \\ Marianella Ortiz-Montes ${ }^{\dagger}$ \\ Santiago Gómez-Echeverry $\ddagger^{\ddagger}$ \\ Andrés F. Rengifo§
}

\section{Resumen}

Según estudios de control social, existe una relación positiva entre las señales de desorden físico y social. En este estudio se utiliza un instrumento de observación directa del entorno en un grupo de colegios de Colombia para estimar la relación de los elementos del entorno físico y social y, la prevalencia de conductas e interacciones no convencionales dentro de estos. Los resultados muestran que a mayores señales de desorden físico, mayor número de conductas e interacciones no convencionales. Sin embargo, se encontró una

* Especialista en Educación, Banco Interamericano de Desarrollo (BID). ORCID: https://orcid.org/0000-0002-9768-0123

† Investigadora, Oficina de Gestión de Proyectos de Investigación-Instituto Colombiano para la Evaluación de la Educación (Icfes). Email: marianella04@hotmail.es ORCID: https://orcid.org/0000-0001-5154-5105

$\ddagger \quad$ Investigador, Oficina de Gestión de Proyectos de Investigación-Instituto Colombiano para la Evaluación de la Educación (Icfes). ORCID: https://orcid.org/0000-0003-30730357

$\S$ Profesor asociado, School of Criminal Justice-Rutgers University, Nueva Jersey. ORCID: https://orcid.org/0000-0003-3345-5055

Para citar este artículo: Dueñas Herrera, X., Ortiz-Montes, M., Gómez-Echeverry, S., \& Rengifo, A. F. (2021). La ecología de las instituciones educativas: entorno, control social y comportamiento en colegios de Colombia . Revista de Economía del Rosario, 24(1), 45-78. https://doi.org/10.12804/revistas.urosario.edu.co/economia/a.10023 
menor prevalencia de estos comportamientos en situaciones de mejor higiene y menos conductas no convencionales en el exterior del colegio.

Palabras clave: control social; desorden; entornos educativos; sociología de la educación. Clasificación JEL: A14, I20, I29, I31.

\title{
The Ecology of Educational Institutions: Environment, Social Control and Behavior in Colombian Schools
}

\begin{abstract}
According to studies of social control, there is a positive relationship between physical, and social disorder. In this study, we employ an onsite observation instrument of the environment to a set of schools in Colombia to estimate the relationship between the physical and social environment elements and the prevalence of non-traditional behaviors and interactions. Our results indicate that the more the signs of physical disorder, the more the number of non-traditional behaviors and interactions. However, we found less prevalence of these behaviors when there are better hygiene and less non-traditional behaviors outside schools.
\end{abstract}

Keywords: Sociology of education; educational environments; disorder; social control. JEL Classification: A14, I20, I29, I31.

\section{A ecologia das instituições educativas: ambiente, controle social e comportamento em colégios da Colômbia}

\author{
Resumo
}

De acordo com estudos de controle social, existe uma relação positiva entre os sinais de desordem físico e social. Neste estudo, usamos um instrumento de observação direta do ambiente em um grupo de escolas colombianas para estimar a relação entre os elementos do ambiente físico e social e a prevalência de comportamentos e interações não convencionais dentro dos colégios. Nossos resultados mostram que quanto maiores os sinais de desordem físico, maior o número de comportamentos e interações não convencionais. No entanto, encontramos uma prevalência menor desses comportamentos devido a uma melhor higiene e menos comportamentos não convencionais fora da escola.

Palavras-chave: sociologia da educação; ambientes educativos; desordem; controle social. Classificação JEL: A14, I20, I29, I31. 


\section{Introducción}

Existe evidencia de un vínculo estrecho entre ciertas señales de deterioro del entorno físico y comportamientos sociales que se consideran indeseados por la sociedad (Sampson \& Raudenbush, 1999; Plank et al., 2009). Por ejemplo, estudios han demostrado que, en los lugares con mayor número de señales de desorden físico, medido a través de variables como los desechos al aire libre y el deterioro generado por el abandono de inmuebles y espacios públicos, se registran mayores niveles de delincuencia, violencia y vandalismo (Shaw \& McKay, 1942; Sampson \& Groves, 1989; Perkins \& Taylor, 1996; Sampson \& Raudenbush, 1999).

Tradicionalmente, esta relación se ha justificado en términos de falta de cohesión y confianza de los residentes de estas áreas, así como ausencia de autorregulación y carencia de mecanismos externos de control (Janowitz, 1975; Sampson et al., 1997). Aunque estas perspectivas se han aplicado regularmente a contextos barriales, son pocas las investigaciones que exploran la relación entre el entorno de las instituciones educativas y los comportamientos de sus estudiantes. Esta perspectiva es importante dado que los colegios son entornos clave de socialización, que a su vez están delineados por varias formas de autoridad, control social y otros recursos de autorregulación.

Teniendo en cuenta lo anterior, en este estudio se aborda la siguiente pregunta de investigación: ¿Qué factores del entorno de los estudiantes pueden explicar sus comportamientos e interacciones? Para responder a este interrogante, se utilizó un instrumento de observación directa del entorno interno y externo en una muestra de colegios públicos y privados $(\mathrm{N}=160)$, seleccionados a conveniencia en diferentes municipios de Colombia. Con esta información, se estimó una serie de modelos de regresión de conteo que relacionan la prevalencia de conductas e interacciones no convencionales entre los estudiantes, entendiendo estas como señales de desorden social, con factores del entorno físico e institucional de los establecimientos educativos.

Para el grupo de instituciones observadas, los análisis indican que ciertas señales de desorden físico, tales como basura o desechos orgánicos en las zonas internas del colegio, están asociadas positivamente con los indicadores de comportamientos no convencionales. Asimismo, los resultados muestran que otras señales del entorno físico de los colegios, particularmente la buena higiene de sus instalaciones — medida de manera subjetiva-, se relaciona de forma negativa con el desorden social observado en el interior de estos (i.e. conductas e interacciones no convencionales).

Dichos hallazgos son importantes dado que, a nuestro conocimiento, son los primeros a nivel latinoamericano que documentan variación en medidas 
de entorno físico y social de colegios y sus áreas más próximas, lo cual representa un aporte que puede complementar otros indicadores de calidad de clima escolar e infraestructura. Adicionalmente, se empleó un instrumento de observación del entorno, que provee una medida más precisa del ambiente en los colegios que las encuestas de percepción que se utilizan usualmente en este tipo de estudios (Raudenbush \& Sampson, 1999).

Dicho lo anterior, este documento se encuentra organizado de la siguiente manera: la segunda sección presenta el marco teórico que relaciona el control social y la regulación de ciertos espacios con medidas de entorno, en el cual está basado el documento. La tercera sección muestra evidencia empírica reciente con un énfasis particular en los contextos educativos y en las contribuciones a nivel regional. La cuarta sección relaciona estas contribuciones con la pregunta de investigación e hipótesis y, explica de manera detallada los instrumentos empleados, los datos recolectados y el método empírico desde el cual se aborda el problema. La quinta sección expone los principales resultados del estudio. Por último, la sexta sección da una serie de conclusiones con base en los ejercicios realizados.

\section{Marco teórico}

Con el fin de entender el desorden en los colegios, se parte de su definición empleada en los análisis a nivel de barrio. Esta definición se fundamenta en la teoría de control social, abordada con amplitud en la sociología y criminología, sustentada en los conceptos establecidos por Thomas Hobbes en el Leviatán. Según esta, las relaciones sociales se encuentran mediadas por acuerdos, contratos y valores que los individuos aceptan e internalizan con el fin de hacer parte de una sociedad, comprometiéndose a no emplear comportamientos perjudiciales para las demás personas. Entonces, se puede entender el control social como la autoridad que ejercen las comunidades sobre sus miembros, mediante instituciones formales e informales, conducente a que estos no transgredan las normas sociales.

El concepto de desorden se ha refinado con el tiempo debido a críticas bien fundamentadas realizadas desde su uso en la literatura. Es de destacar la ambigüedad en la definición del desorden (Skogan, 1990; Kubrin, 2008) y la inadecuada distinción entre el crimen y el desorden por parte de los ciudadanos (Gau \& Pratt, 2008).

Ante esas críticas se han presentado delimitaciones del concepto de desorden tales como la distinción entre el desorden físico y social (Sampson \& Raudenbush, 1999; Chappell et al., 2011). De modo que, en múltiples estudios el desorden físico es definido como el deterioro observado en un entorno 
físico particular - e.g. grafitis en los edificios, carros abandonados y basura en las calles- mientras que el desorden social, comúnmente denominado incivilidades, es entendido como aquellos comportamientos que pueden considerarse peligrosos o amenazantes, como el acoso verbal, la prostitución o los grupos tumultuosos de jóvenes en las calles. Estas señales, por tanto, pueden ser el reflejo de la falta de control social en la comunidad (Ross \& Mirowsky, 1999).

Esta distinción teórica, ya se encontraba latente en trabajos empíricos como el realizado con la teoría de las ventanas rotas (Wilson \& Kelling, 1982), el cual vincula estas dos medidas de desorden. Según dicha teoría, las señales visibles de desorden físico, como los vidrios rotos o las basuras al aire libre, pueden promover que los individuos incurran en un ciclo de trasgresiones al orden convencional (i.e. desorden social o incivilidades) debido a dos mecanismos diferentes.

Por un lado, el desorden puede hacer que los habitantes de la zona la perciban como peligrosa $\mathrm{y}$, dado el miedo que esto les genera, terminen ejerciendo un menor control social en esta. Por otro lado, el desorden puede hacer que los posibles transgresores vean la falta de orden como una señal de control social débil y, con ello, perciban una menor probabilidad de ser sancionados por sus conductas (véase Keuschnigg \& Wolbring, 2015). Esto los llevará a realizar con mayor frecuencia actividades no convencionales o, incluso, delictivas. Las relaciones entre las variables establecidas con estos dos mecanismos se encuentran ilustradas en la figura 1 .

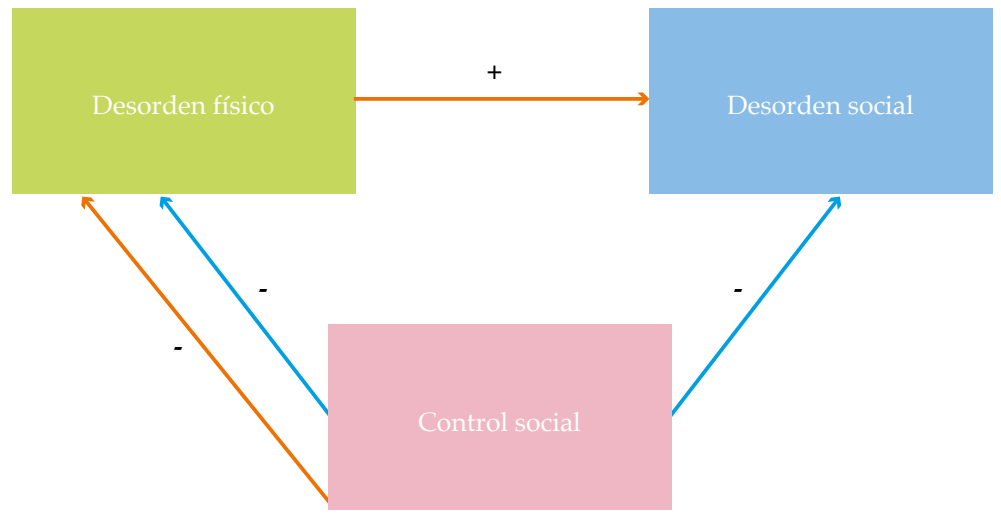

Figura 1. Desorden físico y desorden social según la teoría de las ventanas rotas Nota: modelo conceptual de las ventanas rotas. Las líneas azules denotan el primer mecanismo de relación entre el desorden físico y el desorden social planteado; mayores señales de desorden físico redundan en menor control social de los habitantes, y por tanto, en mayor desorden social. Las líneas naranjas denotan el segundo mecanismo mencionado.

Fuente: elaboración propia a partir de Lanfear et al. (2020). 
Esta teoría ha sido el centro de desarrollo de múltiples políticas públicas de seguridad en diversos lugares del mundo, siendo ampliamente discutida y aplicada en Estados Unidos. Aún con esto, no es claro si la relación causal entre el desorden físico y el desorden social que establece esta corriente teórica, es el verdadero mecanismo que lleva al deterioro zonal, o si en realidad, el desorden físico y social son producto de la falta de control social en la zona, tal y como lo establece la teoría del desorden social (Shaw \& McKay, 1931, 1942; Kondo et al., 2018; Lanfear et al., 2020).

Por tanto, este estudio busca hacer un aporte a la literatura del desorden social mediante la aplicación de sus conceptos clave en el contexto educativo colombiano, aproximación que, como se ha mencionado, no se ha realizado antes.

\section{Evidencia empírica}

\subsection{Contexto barrial}

La relación entre el desorden físico e indicadores de desorden social, tales como las tasas de crímenes, ha sido abordada ampliamente en la criminología. En estos estudios hay un debate constante producto de dos posiciones: quienes mantienen que los crímenes son generados principalmente por características individuales (Lilly et al., 2002); y quienes sostienen que las características y procesos macro son los que guían la dispersión del crimen en las ciudades (Shaw \& McKay, 1942).

Con el fin de aportar a esta discusión y fomentar una visión ecológica en el análisis criminológico que estuvo ausente durante el final del siglo pasado (Messner, 1988), Pratt y Cullen (2005) desarrollaron un meta-análisis de la relación entre el desorden y el crimen con el fin de evaluar cuáles son las variables que mejor predicen los delitos y, de esta manera, valorar las diferentes teorías ecológicas del crimen. Sus resultados muestran que las variables que permiten obtener una mejor estimación del crimen son aquellas que indican alta concentración de vulnerabilidad (i.e. pobreza y rupturas familiares), y que con esto, las teorías explicativas del crimen que mayor soporte empírico tienen, son aquellas que se centran en el desorden social y en la de privación económica. Debido a estos resultados es que esta investigación se concentrará en esas corrientes teóricas para el análisis de los datos.

En esa perspectiva, Brown et al. (2004) también encuentran que el desorden físico es un buen predictor del crimen. En su estudio, los autores emplean información censal e inventarios del entorno en Salt Lake City (Estados Unidos) para realizar un análisis mediante modelos jerárquicos 
lineales, y, con esto, llegar a la conclusión de que el deterioro físico en los barrios se relaciona positivamente con los crímenes reportados por la policía. Más aún, evidencian que la percepción de los residentes del barrio no tiene una relación estadística con los crímenes, por lo cual, consideran que valdría la pena evaluar empíricamente el primer mecanismo establecido en la teoría de las ventanas rotas para conectar el desorden físico y social con mayor profundidad.

Así mismo, estudios cuasi-experimentales como los de Branas et al. (2018), han encontrado evidencia acerca del efecto que tiene el desorden sobre el crimen. ${ }^{1}$ Su experimento, realizado en Estados Unidos, consistía en limpiar el desorden físico que se encontraba alrededor de lotes baldíos en horarios fijos, creando una atmósfera distinta en las áreas intervenidas. Los resultados muestran que su intervención redujo las percepciones sobre el crimen (37\%), vandalismo (39\%) y, ciertos comportamientos de las personas, como el quedarse en la casa por motivos de seguridad (58\%). Su experimento también mostró efectos positivos en la socialización de las personas por fuera de su hogar $(76 \%)$.

Si bien esta relación se ha analizado más frecuentemente en Estados Unidos y Europa (e.g. Keuschnigg \& Wolbring, 2015; Berger \& Hevenstone, 2016), existen múltiples estudios que han detallado el vínculo entre el desorden físico y social en América Latina. Villareal y Silva (2006) examinaron los efectos de la cohesión social y el desorden de los barrios sobre el crimen en Belo Horizonte (Brasil) empleando información de la Encuesta de Victimización de Belo Horizonte, realizada por la Universidad de Minas Gerais en el 2002, por medio de una serie de modelos multinivel. Sus análisis mostraron que, contrario a lo que ocurre en Estados Unidos y Europa, los barrios más vulnerables de la ciudad contaban con mayores niveles de cohesión social, pero a su vez, tenían mayores tasas de victimización. Además, los autores encontraron una relación positiva y estadísticamente significativa entre el desorden físico y social de los barrios y, el número de asaltos y homicidios cometidos en ellos.

Por su parte, Cerdá et al. (2012) analizaron los efectos de las tasas de crímenes en una intervención realizada por el Gobierno local de Medellín (Colombia), en la cual, junto con la implementación de un sistema de transporte urbano, se ejecutaron una serie de mejoras a la infraestructura barrial en zonas vulnerables. Mediante el uso de información longitudinal y modelos lineales jerárquicos generalizados en una muestra emparejada de barrios, los autores encontraron que la intervención generó una reducción del $66 \%$

1 Véase Branas et al. (2016). 
en las tasas de homicidio y disminución del $75 \%$ de la violencia reportada por los ciudadanos.

\subsection{Contexto educativo}

Existen múltiples trabajos que estudian el desorden y el control social en el contexto educativo desde un punto de vista ecológico. ${ }^{2}$ Plank et al. (2009), por ejemplo, emplearon datos de 33 colegios públicos pertenecientes a un distrito escolar en Atlantic City (Estados Unidos) en diferentes modelos de ecuaciones estructurales con el objetivo de relacionar el desorden físico, el miedo, la eficacia colectiva y el desorden social. Los autores encuentran una relación directa entre el desorden físico - medido a través de variables como la limpieza de los edificios o el número de ventanas, puertas o escritorios rotos- y el desorden social - medido a través de indicadores de problemas como abuso físico o verbal de docentes y peleas de estudiantes-, aun controlado por la eficacia colectiva previa. De acuerdo con sus resultados, el desorden físico es un factor relevante a la hora de analizar las percepciones y el comportamiento de los estudiantes y el clima escolar.

Por otro lado, Uline y Tschannen-Moran (2008) emplearon encuestas con docentes y los resultados de pruebas estandarizadas de los estudiantes de 80 colegios en Virginia (Estados Unidos), con el fin de examinar el vínculo entre la calidad estructural de los colegios con (i) las actitudes, comportamientos de los docentes y estudiantes, y (ii) el desempeño académico de estos últimos. El análisis exploratorio de los autores, que empleó métodos correlacionales y de regresión, mostró que el deterioro en la estructura de los colegios se relaciona negativamente con el involucramiento de la comunidad educativa, las actitudes de los docentes y el desempeño de los estudiantes. Aún más, los resultados evidencian que el clima escolar es un mediador de la relación entre el deterioro físico de los colegios y el desempeño académico.

Como complemento de este análisis, Uline y Tschannen-Moran (2008) tomaron 2 de los 80 colegios de su anterior trabajo para realizar un estudio de caso con el fin de analizar cómo el entorno escolar afectaba el ambiente de aprendizaje. Estos colegios fueron seleccionados dadas las altas valoraciones de la infraestructura que recibieron por parte del personal y, puesto que en ellos, más del $50 \%$ de los estudiantes recibía descuentos de alimentación. Usando la información recolectada con grupos focales, recorridos por los colegios y documentación fotográfica, los autores concluyeron que hay

2 Véase Uline y Tschannen-Moran (2008), Plank et al. (2009), Braham (2004) y Mijanovich y Weitzman (2003). 
una relación entre el entorno físico de los establecimientos y las medidas de aprendizaje y enseñanza. También, encontraron que la interacción entre el compromiso de la comunidad educativa y el estado de las instalaciones del colegio afecta de manera considerable el ambiente de aprendizaje.

Si bien existe un considerable número de estudios que abordan el problema enfocándose en el contexto educativo, tal y como se ha mostrado, son pocos los que emplean observaciones directas de las instituciones educativas en Latinoamérica (Wilcox et al., 2006). Dicho lo anterior, con este estudio se pretende aportar a la literatura con una exploración detallada del entorno escolar que no se ha realizado previamente en el contexto colombiano.

\section{Datos}

\subsection{Bases de datos}

Los datos empleados fueron recolectados en septiembre del 2017, en paralelo con la aplicación de la prueba Saber 3. $5^{\circ} .^{\circ}$ y $9 .{ }^{\circ},{ }^{3}$ realizada por el Instituto Colombiano para la Evaluación de la Educación (Icfes), a través de un instrumento de observación sistemática del entorno escolar en un conjunto de sedes-jornadas ${ }^{4}$ en Colombia.

La prueba Saber 3. , $^{\circ} .^{\circ}$ y $9 .^{\circ}$ tiene dos modalidades de aplicación: (i) autoaplicada y (ii) muestra controlada. La modalidad auto-aplicada, como su nombre lo indica, se caracteriza porque los colegios son quienes se encargan de la aplicación de la prueba con apoyo logístico del Icfes. Y en la modalidad controlada, se selecciona una muestra representativa del universo de colegios con el fin de que el Icfes monitoree aspectos de implementación de la prueba, calibre preguntas en adaptación y realice el pilotaje de nuevas preguntas. Aunque todos los colegios de esta muestra tienen un acompañamiento del Icfes, un conjunto de estas instituciones cuenta con un apoyo adicional para la prueba. Dicho acompañamiento está a cargo de funcionarios (monitores) que se aseguran de que el proceso de seguimiento a los colegios coordinado

3 La prueba Saber es un examen estandarizado que presentan todos los estudiantes de colegios públicos y privados de los grados $3 .^{\circ}, 5^{\circ}$ y $9 .^{\circ}$ en Colombia, que tiene por objetivo evaluar sus competencias en las áreas de matemáticas y lenguaje.

4 Las sedes son plantas físicas independientes que conforman una institución educativa y son administradas en su totalidad, por un establecimiento educativo principal. La jornada escolar es el tiempo que dedica una sede a la prestación directa del servicio educativo, puede darse de cuatro formas: completa, mañana, tarde y sabatina. Este estudio se enfocó en las sedes-jornadas mañana y tarde con el fin de maximizar el número de colegios a visitar. 
por el Icfes sea el adecuado. Esta característica particular de la aplicación controlada, permitió la implementación del instrumento de observación del entorno en el conjunto mencionado.

La selección de las sedes-jornadas en la aplicación controlada fue realizada por la Subdirección de Estadísticas del Icfes utilizando un muestreo estratificado bietápico, aplicado a la muestra de establecimientos educativos registrados en Colombia. Para ello, se consideraron tres estratos según la zona y el sector al que las instituciones pertenecen: (i) Oficial-Rural, (ii) OficialUrbano y (iii) No Oficial. Asimismo, se seleccionaron los establecimientos educativos con base en una probabilidad de inclusión proporcional a la matrícula total en los grados de interés.

A partir de esa muestra, se seleccionó a conveniencia un grupo de entidades educativas para el monitoreo y la realización de las observaciones del entorno escolar. La decisión de cuántas instituciones visitar se tomó considerando la disponibilidad de los funcionarios del Icfes seleccionados para ser monitores, con el objetivo de maximizar el número de colegios por observador. Por esta razón, se solicitó a cada uno de los funcionarios que visitara 4 sedes-jornadas en la misma ciudad y, en la medida de lo posible, con una ubicación relativamente cercana para recolectar la mayor cantidad posible de información.

Lo anterior dio como resultado un conjunto de 160 sedes-jornadas para observación por parte de 55 funcionarios del Icfes. Estos monitores fueron debidamente capacitados para diligenciar los instrumentos de observación. ${ }^{5}$ Cada monitor observó en promedio 3 colegios, cada uno en un día, con un tiempo aproximado de observación de 30 minutos por instrumento. Las visitas se realizaron en un espacio de 3 días.

Teniendo en cuenta que el ejercicio de observación no podía afectar las demás funciones que los monitores debían llevar a cabo el día de la aplicación de la prueba, se les dio vía libre para que realizaran las observaciones en la hora y día que encontraran más conveniente dentro del rango de tiempo permitido. Todos los instrumentos de observación fueron cargados en la plataforma Qualtrics y su diligenciamiento se llevó a cabo a través de dispositivos móviles.

5 La capacitación brindada a los funcionarios consistió en una explicación detallada de cada uno de los ítems a responder con base en la observación del entorno, haciendo especial énfasis en aquellas preguntas que podían prestarse para confusiones como el conteo de grafitis en las paredes. Asimismo, se les entregó un manual de observación en el cual se precisó la forma adecuada de responder cada pregunta junto con imágenes ilustrativas de posibles escenarios. 
Es necesario aclarar que la aplicación de la prueba Saber $3 .^{\circ}, 5 .^{\circ}$ y $9 .^{\circ}$, se hace en semana escolar y, a pesar de que gran parte del colegio está en función del examen, los grados que no son evaluados tienen un día normal de clase. Esto es relevante ya que permite capturar dinámicas sociales de un día regular de colegio y reducir considerablemente sesgos de comportamiento inducidos por el hecho de ser observados (i.e. características de demanda). Sin embargo, la presentación de una prueba de estado puede afectar considerablemente el comportamiento de los estudiantes, el personal administrativo y el personal docente, en lo referente al orden del colegio - e.g. las personas no arrojan basuras a su alrededor y los profesores están más pendientes del comportamiento de sus estudiantes ese día que en uno normal—.

\subsection{Instrumentos}

Se aplicaron dos instrumentos de observación del entorno escolar al conjunto de sedes-jornada. Uno para el interior de los colegios y otro para su exterior. Estos instrumentos se construyeron a partir de los trabajos realizados por Sampson y Raudenbush (1999), Milam et al. (2010), Plank et al. (2009) y Bradshaw et al. (2015), cuyo enfoque principal ha sido la observación del entorno de los barrios para detectar señales de desorden físico, social y sus posibles consecuencias en el miedo, la eficacia colectiva y el crimen.

El instrumento para el interior de la sede-jornada medía aspectos como el desorden físico, el desorden social, la higiene y el ambiente de inseguridad en el colegio. Durante su recorrido, los observadores debían registrar si encontraban en las diferentes zonas del colegio elementos como basura/desperdicios por fuera de las canecas, paredes y/o techos deteriorados, colillas de cigarrillo, grafitis, lavamanos en mal estado, cámaras de seguridad, entre otros.

De la misma manera, para capturar el entorno social, se les pidió a los observadores que registraran los casos en los que observaran estudiantes tatuados, portando armas, peleando, realizando demostraciones excesivas de afecto y demás (véase en los anexos tabla 4).

Por otra parte, los aspectos medidos al exterior de los colegios fueron muy similares a los del interior, considerando la posibilidad de encontrar mayor heterogeneidad en estos contextos. Así, se realizaron adaptaciones para incluir la observación de huecos en la calle, lotes abandonados, caños, tiros en las paredes, vendedores ambulantes e instituciones estatales, en los alrededores del colegio. Adicionalmente, se capturaron variables comportamentales en las cercanías del colegio con registros como el número de jóvenes deambulando por la calle, personas consumiendo drogas, personas peleando o gritando, entre otros comportamientos (véase en los anexos tabla 5). 
A partir de la información de estos instrumentos, se construyó un total de nueve variables de conteo: (i) conductas no convencionales; (ii) interacciones sociales no convencionales; ${ }^{6,7}$ (iii) señales de desorden físico dentro del colegio; (iv) baños con problemas; (v) ambiente de inseguridad; (vi) higiene objetiva; (vii) higiene subjetiva; (viii) conductas no convencionales por fuera del colegio y (ix) señales de desorden físico por fuera del colegio. Las variables mencionadas se construyeron a partir de la suma de variables dicótomas que se reconocen en la literatura comúnmente como medidas de desorden. En las tablas 4 y 5 de los anexos se presentan cada uno de los aspectos nombrados junto con los ítems que los componen.

Tal y como se puede ver en la tabla 4 de los anexos, las conductas no convencionales se capturaron mediante el registro de observaciones por colegio de: (i) estudiantes con perforaciones, pelo de colores, uñas pintadas, tatuajes y no uniformados; (ii) estudiantes embarazadas; (iii) estudiantes con armas -e.g. cuchillos, elementos cortopunzantes, armas de fuego- ${ }^{8}$ y (iv) estudiantes fumando. De otra parte, el conteo de interacciones no convencionales se creó agrupando los ítems de: (i) estudiantes peleando o discutiendo acaloradamente; (ii) demostraciones de afecto excesivas; (iii) comunicación entre estudiantes y personas externas a la comunidad educativa e (iv) intercambio de materiales, artefactos $u$ otros elementos entre estudiantes y personas externas al colegio.

Asimismo, se crearon variables de conteo empleadas como explicativas en el análisis. El conteo de señales de desorden físico por dentro del colegio, se hizo a partir de la suma de casos en que el aplicador observó elementos tales como animales merodeando dentro de la institución, excremento, drogas, colillas de cigarrillos, entre otros. El ambiente de inseguridad se midió por el

6 Las conductas e interacciones se catalogaron como no convencionales siguiendo la definición de convención dada por Max Weber, según la cual: “Convención debe llamarse a la 'costumbre' que, dentro de un círculo de hombres se considera como válida y que está garantizada por la reprobación de la conducta discordante" (Weber, 1964, p. 27). De igual manera, estas se basan en lo establecido por las normas morales, las cuales indican de forma general lo que una comunidad espera de sus miembros en ciertas áreas particulares de la vida social, en este caso, de los colegios y lo que se consideran rechazos a estas expectativas (Stebbins, 2011).

7 Si bien es cierto que dada su estructura esta variable se asemeja a lo que se denomina incivilidades en la teoría de desorden en los barrios, se decidió no emplear esta denominación en vista de que ese concepto presenta variaciones en el contexto educativo.

8 Entendiendo que el hecho de observar estudiantes con armas puede tener una connotación diferente a las demás variables utilizadas en la construcción de este conteo, se consideró un análisis de sensibilidad para ver cómo este hecho podía afectar los resultados. 
conteo de señales como ventanas y puertas con barrotes, cámaras de seguridad, trozos de vidrios en los muros, etcétera. El aspecto de higiene se capturó a través de un conteo de deshechos que daba la percepción objetiva de este factor $y$, una serie de valoraciones subjetivas realizadas por los observadores en los diferentes espacios del colegio. ${ }^{9}$

Finalmente, las conductas no convencionales por fuera del colegio se midieron con el conteo de casos en que los observadores notaron señales como jóvenes deambulando, personas consumiendo algún tipo de droga, personas tomando alcohol, etcétera. Asimismo, el desorden físico por fuera del colegio se midió mediante el conteo de lotes abandonados, aceras rotas, tiros en las paredes, entre otros.

\section{Análisis}

\subsection{Estadísticas descriptivas}

La tabla 1 muestra las estadísticas descriptivas de cada uno de los rasgos del entorno observados y de una serie de características sociodemográficas de los colegios. En el Panel a se presentan las variables de conteo empleadas como dependientes en las estimaciones — que se encuentran en cursivajunto con los ítems que la componen. Los aspectos de interés se encuentran en el Panel b, los cuales son: (i) señales de desorden físico dentro del colegio, (ii) ambiente de inseguridad, (iii) higiene subjetiva, (iv) higiene objetiva, (v) problemas en los baños, (vi) señales de conductas no convencionales por fuera del colegio y (vi) señales de desorden físico fuera del colegio. Por último, en el Panel c se muestran las características de los establecimientos que se incluyeron como variables de control en los análisis.

Tabla 1. Estadísticas descriptivas

\begin{tabular}{|c|c|c|c|c|c|c|}
\hline Variable & $\begin{array}{l}\text { N. obser- } \\
\text { vaciones }\end{array}$ & Promedio & $\begin{array}{l}\text { Desviación } \\
\text { estándar }\end{array}$ & Mínimo & Máximo & $\begin{array}{l}\text { Variables } \\
\text { Discretas }\end{array}$ \\
\hline \multicolumn{7}{|l|}{ Panel a } \\
\hline $\begin{array}{l}\text { Conteo de conductas no } \\
\text { convencionales }\end{array}$ & 157 & 0.242 & 0.624 & 0 & 4 & 0.178 \\
\hline $\begin{array}{l}\text { Estudiantes con piercings, } \\
\text { pelo de colores, uñas pinta- } \\
\text { das, tatuajes, no uniformados }\end{array}$ & 157 & 0.159 & & 0 & 1 & \\
\hline
\end{tabular}

9 La variable de higiene subjetiva se construyó sumando el número de espacios en los que el observador calificó la higiene como buena o muy buena. 


\begin{tabular}{|c|c|c|c|c|c|c|}
\hline Variable & $\begin{array}{l}\text { N. obser- } \\
\text { vaciones }\end{array}$ & Promedio & $\begin{array}{l}\text { Desviación } \\
\text { estándar }\end{array}$ & Mínimo & Máximo & $\begin{array}{l}\text { Variables } \\
\text { Discretas }\end{array}$ \\
\hline Estudiantes embarazadas & 157 & 0.051 & & 0 & 1 & \\
\hline Estudiantes fumando & 157 & 0.006 & & 0 & 1 & \\
\hline $\begin{array}{l}\text { Estudiantes con armas (por } \\
\text { ejemplo, cuchillos, elemen- } \\
\text { tos cortopunzantes, armas) }\end{array}$ & 157 & 0.025 & & 0 & 1 & \\
\hline $\begin{array}{l}\text { Conteo de interacciones so- } \\
\text { ciales no convencionales }\end{array}$ & 157 & 0.35 & 0.715 & 0 & 3 & 0.229 \\
\hline $\begin{array}{l}\text { Estudiantes peleando o dis- } \\
\text { cutiendo acaloradamente }\end{array}$ & 157 & 0.096 & & 0 & 1 & \\
\hline $\begin{array}{l}\text { Demostraciones de afecto } \\
\text { excesivas entre estudiantes }\end{array}$ & 157 & 0.096 & & 0 & 1 & \\
\hline $\begin{array}{l}\text { Comunicación entre } \\
\text { estudiantes y externos }\end{array}$ & 157 & 0.115 & & 0 & 1 & \\
\hline $\begin{array}{l}\text { Intercambio entre } \\
\text { estudiantes y externos }\end{array}$ & 157 & 0.045 & & 0 & 1 & \\
\hline \multicolumn{7}{|l|}{ Panel b } \\
\hline $\begin{array}{l}\text { Conteo de señales de } \\
\text { desorden físico dentro } \\
\text { del colegio }\end{array}$ & 157 & 2.013 & 1.437 & 0 & 6 & 0.828 \\
\hline $\begin{array}{l}\text { Conteo de ambiente de } \\
\text { inseguridad }\end{array}$ & 157 & 3.038 & 1.4 & 0 & 6 & 0.955 \\
\hline Conteo de higiene subjetiva & 157 & 5.752 & 1.576 & 0 & 7 & 0.981 \\
\hline Conteo de higiene objetiva & 157 & 1.981 & 0.178 & 0 & 2 & 0.994 \\
\hline $\begin{array}{l}\text { Conteo de problemas en } \\
\text { los baños }\end{array}$ & 157 & 2.127 & 0.979 & 0 & 5 & 0.911 \\
\hline $\begin{array}{l}\text { Conteo de señales no } \\
\text { convencionales por fuera } \\
\text { del colegio }\end{array}$ & 148 & 1.418 & 1.591 & 0 & 7 & 1.000 \\
\hline $\begin{array}{l}\text { Conteo de señales de desor- } \\
\text { den físico afuera del colegio }\end{array}$ & 148 & 5.426 & 3.35 & 0 & 16 & 0.943 \\
\hline \multicolumn{7}{|l|}{ Panel c } \\
\hline Índice de ruralidad ${ }^{10}$ & 157 & 0.067 & 0.092 & 0.001 & 0.473 & \\
\hline $\begin{array}{l}\text { Logaritmo natural del } \\
\text { número de estudiantes } \\
\text { en la sede-jornada }\end{array}$ & 157 & 6.178 & 1.039 & 2.833 & 8.253 & \\
\hline
\end{tabular}

Continúa

10 El índice de ruralidad está construido como la fracción entre la población rural y total. 


\begin{tabular}{lccccc}
\hline \multicolumn{1}{c}{ Variable } & $\begin{array}{c}\text { N. obser- } \\
\text { vaciones }\end{array}$ & Promedio & $\begin{array}{c}\text { Desviación } \\
\text { estándar }\end{array}$ & Mínimo Máximo & $\begin{array}{c}\text { Variables } \\
\text { Discretas }\end{array}$ \\
\hline $\begin{array}{l}\text { Nivel Socioeconómico (INSE) } \\
\text { del establecimiento }\end{array}$ & 157 & 52.46 & 5.311 & 38.109 & 70.918 \\
$\begin{array}{l}\text { Número de sedes } \\
\begin{array}{l}\text { Colegio con afiliación reli- } \\
\text { giosa explícita/observable }\end{array}\end{array}$ & 157 & 1.924 & 1.029 & 1 & 5 \\
$\begin{array}{l}\text { Oficial } \\
\begin{array}{l}\text { Observador hombre } \\
\hline\end{array}\end{array}$ & 157 & 0.439 & 0.834 & 0 & 1 \\
\hline
\end{tabular}

Nota: todos los análisis se realizaron con una muestra de 157 colegios de los 160 que fueron seleccionados originalmente. Los 3 tres colegios que no se incluyeron en el conjunto final fueron excluidos debido a que en estos no se contaba con suficiente información sobre el entorno educativo.

Para las variables de conteo aquí presentadas, adicional a las estadísticas descriptivas, se incluyó el valor esperado de su transformación dicotómica (i.e. 0 sin evento, 1 con al menos un evento observado) en la última columna de la tabla.

Fuente: elaboración y cálculos propios a partir de información del Icfes, Ministerio de Educación Nacional y panel municipal del Centro de Estudios sobre Desarrollo Económico - cEDE de la Universidad de los Andes.

Los datos indican que en el conjunto de colegios hay más interacciones sociales no convencionales ( 0.35 eventos), que conductas no convencionales (0.24 eventos). Además, con la información del Panel a se nota que la conducta no convencional más frecuente fue el porte de piercings, pelo de colores, uñas pintadas, tatuajes y/o no uniformados (0.16 eventos). De la misma manera, la interacción social no convencional que predominó en el conjunto de colegios fue la comunicación entre estudiantes y personas externas a la institución educativa (0.11 eventos).

En general, se observan pocas señales de desorden físico o de baños con problemas. Referente al ambiente de inseguridad, dado que las principales medidas de tendencia central - e.g. media, moda y mediana - en los colegios toman un valor cercano a 3 y el rango de esta variable es de 0 a 6 , no es posible asegurar que las instituciones observadas sean particularmente seguras o inseguras. A su vez, se encontró que los colegios tienen buena higiene, tanto de forma objetiva como subjetiva, dada la posición de los promedios dentro de los rangos de resultado. Asimismo, los observadores reportaron haber visto un bajo número de conductas no convencionales alrededor de los colegios (promedio 1.4 de 7 posibles comportamientos), tales como la presencia de personas consumiendo alcohol o discutiendo acaloradamente (véase en los anexos tabla 5). 
Con la información del panel c se muestra que los colegios del análisis son en su mayoría oficiales ( $83 \%$ ), de nivel socioeconómico medio (52.5 en una escala de 0 a 100), cuentan en promedio con un número de estudiantes mayor que la media nacional (i.e. 151 estudiantes por sede-jornada de acuerdo con la información del Sistema Integrado de Matrícula —SIMAT-, 5.02 en logaritmo natural), se encuentran ubicados en zonas urbanas y, que cerca de la mitad (44\%) de las instituciones educativas tiene algún tipo de afiliación religiosa. Por cierto, es de resaltar que el $61 \%$ de las personas que diligenciaron los instrumentos fueron hombres, por lo cual en la muestra se contó con una visión predominante de este sexo. ${ }^{11}$

Previo a realizar los análisis de regresión, se exploraron las relaciones entre las variables de resultado y las variables de interés por medio de correlaciones de Spearman. Con esto se tiene evidencia de que las conductas no convencionales tienen una relación inversa con la medida de higiene subjetiva $(-0.26)^{12}$ y una relación positiva con el desorden físico dentro del colegio (0.24). Al analizar las interacciones sociales no convencionales se encontró una relación estadísticamente significativa con las conductas no convencionales por fuera del colegio (0.26) y con las señales de desorden físico (0.22).

En cuanto a la relación existente entre las variables de resultado y los controles, se evidenció que la única relación estadísticamente significativa es la del sexo del observador (-0.17 y 0.24 para conductas e interacciones no convencionales, respectivamente). Finalmente, la relación más fuerte y estadísticamente significativa entre las variables de interés, se da entre el índice de ruralidad y nivel socioeconómico de la institución educativa (-0.47).

\subsection{Análisis multivariado}

En vista de que las variables dependientes son conteos de eventos, lo más adecuado para el análisis es emplear métodos de regresión que reconozcan las distribuciones de este tipo de variables. Más aún, si se ignoraran estas distribuciones y emplearan regresiones por Mínimos Cuadrados Ordinarios (мСO), se estarían usando métodos en los cuales los supuestos no son válidos - e.g. normalidad de los errores - y, se podrían encontrar valores estimados negativos. Lo último no tiene sentido en este caso, ya que se está tratando

11 Se entiende que esta variable de control, si bien no es parte del enfoque principal de este documento, es fundamental para el modelo de análisis en vista de las diferencias existentes en la percepción de riesgo entre sexos (Logan \& Walker, 2017).

12 Todas las relaciones mencionadas son significativas a los niveles convencionales, es decir, al $10 \%, 5 \%$ o $1 \%$. 
con conteos y, por ende, la variable dependiente debe encontrarse dentro del conjunto de número naturales y los estimados dentro del conjunto de números reales positivos (Cameron \& Trivedi, 1998).

Por lo anterior, se emplearon regresiones de conteo las cuales reconocen adecuadamente las distribuciones asimétricas que tienen las variables de resultado. En todos los modelos se asumió que la variable dependiente sigue una distribución Poisson, de manera que la distribución de esta puede representarse como:

$$
\operatorname{Pr}\left(y_{i}\right)=\frac{e^{-\mu_{i}} * \mu_{i}^{y_{i}}}{y_{i} !}
$$

Donde $y_{i}$ es la variable de conteo que se toma como resultado y $\mu_{i}$ es el parámetro de intensidad o el número de veces que se espera que ocurran los eventos determinados. Este parámetro se estima mediante la función:

$$
\mu_{i}=E\left(y_{i} \mid x_{i}\right)=\exp \left(x_{i}^{\prime} \beta+w_{i}^{\prime} \gamma\right)
$$

Para este caso específico, el término $x_{i}$ representa el conjunto de variables independientes que captura los aspectos de interés y $\beta$ es un vector de coeficientes que mide las relaciones entre estas variables y las medidas de desorden social. Por otra parte, en el vector $w_{i}$ están contenidas todas las variables de control empleadas en el análisis y que capturan características de las sedes-jornada como el índice socioeconómico construido por el Icfes, el número de estudiantes en la sede-jornada que reporta el sIMAT mediante la plataforma buscando colegio y, el índice de ruralidad del municipio construido por el Departamento Administrativo Nacional de Estadística (DANE).

Las tablas 2 y 3 muestran los resultados de las estimaciones de las medidas de desorden social consideradas, que son las conductas no convencionales y las interacciones sociales no convencionales, respectivamente. ${ }^{13}$ Adicional a las variables de interés y de control, se agregaron interacciones entre las señales de desorden físico dentro del colegio y las conductas no convencionales por fuera de él con: (i) la afiliación religiosa, (ii) la cantidad

13 Además de las correlaciones presentadas, con el fin de evaluar posibles problemas de multicolinealidad en las regresiones: (i) se calcularon las correlaciones entre cada par de variables independientes y controles empleando la corrección de Bonferroni, y (ii) se calcularon los factores de inflación de la varianza para cada una de las variables explicativas del modelo. Los resultados de este ejercicio mostraron que no existen problemas de multicolinealidad que comprometan las estimaciones realizadas. 
de estudiantes en la institución y (iii) el índice de ruralidad. Esto con el fin de determinar si esos factores se relacionan de manera conjunta con un incremento o una reducción de las conductas e interacciones no convencionales de los estudiantes.

Se tuvieron en cuenta las variables mencionadas para evaluar términos de interacción debido a su relevancia teórica en esta relación de interés. Por un lado, en vista de que los colegios religiosos tienden a contar con normas de conducta más estrictas que los colegios laicos (Andrade Álvarez, 2011) tiene sentido pensar que esta medida es un moderador relevante. Por otro lado, en vista de que la probabilidad de encontrar conductas no convencionales por fuera del colegio es menor en los colegios que se encuentran en zonas rurales, se consideró que esta medida también debía ser incluida en el análisis. Por último, debido a que ante mayor número de estudiantes en el colegio es más difícil mantener el orden dentro de él, la última inclusión al conjunto de variables moderadoras fue la matrícula de los colegios.

El modelo 1 incluye únicamente los aspectos de interés de desorden físico dentro del colegio, ambiente de inseguridad, higiene subjetiva y objetiva, problemas en los baños, conductas no convencionales y señales de desorden físico por fuera del colegio. Los resultados de este ejercicio indican que el conteo de señales de desorden físico dentro del colegio está relacionado positivamente con el número de conductas no convencionales dentro de este; una señal de desorden físico adicional se asocia con un incremento de $26 \%$ en el número de conductas no convencionales. La significancia estadística de esta relación se mantiene aun cuando se incluyen controles característicos de las sedes-jornada (modelo 2) y los términos de interacción con conductas no convencionales por fuera de estas (modelo 4). También se encontró que, a mayor higiene, medida de forma subjetiva, el número de conductas no convencionales estimadas decrece, relación que se mantiene significativa en todos los modelos exceptuando el 4. Asimismo, a pesar de que en el modelo 1 la relación entre las conductas no convencionales por fuera de los colegios y este mismo tipo de conductas al interior de estos no resulta significativa, en los demás modelos se evidencia que estas se relacionan positivamente.

14 Los coeficientes resultantes de las regresiones Poisson pueden ser interpretados como la diferencia de logaritmos de la variable de resultado conforme cambia la variable independiente en una unidad. Usualmente, esta diferencia se interpreta como el cambio porcentual de la variable dependiente, debido a la expansión de Taylor de primer orden de los logaritmos. Sin embargo, esta equivalencia no se cumple en los casos en los cuales los cambios son considerablemente grandes. Dado esto, y con el fin de obtener el cambio porcentual de la variable dependiente, se transformaron los coeficientes resultantes de la regresión con la función: $\mathrm{f}=\mathrm{e}-1$. 
Finalmente, con respecto a las interacciones entre variables (véase en los anexos figuras 3 y 4 para visualizar los resultados de las interacciones), solo se encontró una relación estadísticamente significativa entre las conductas no convencionales por fuera de los colegios y el índice de ruralidad. Entre más señales de esta medida de desorden social por fuera del colegio haya en zonas más rurales, menores son las conductas no convencionales que se presentan al interior de las instituciones educativas observadas.

Tabla 2. Regresiones Poisson-Conductas no convencionales

\begin{tabular}{|c|c|c|c|c|}
\hline Variables & Modelo 1 & Modelo 2 & Modelo 3 & Modelo 4 \\
\hline $\begin{array}{l}\text { Conteo de señales de desorden físico } \\
\text { dentro del colegio }\end{array}$ & $\begin{array}{l}0.257^{* *} \\
(0.105)\end{array}$ & $\begin{array}{l}0.328^{* *} \\
(0.111)\end{array}$ & $\begin{array}{l}-0.426 \\
(0.614)\end{array}$ & $\begin{array}{c}0.505^{* * *} \\
(0.154)\end{array}$ \\
\hline Conteo de ambiente de inseguridad & $\begin{array}{c}0.033 \\
(0.114)\end{array}$ & $\begin{array}{c}0.006 \\
(0.152)\end{array}$ & $\begin{array}{l}-0.029 \\
(0.141)\end{array}$ & $\begin{array}{l}-0.040 \\
(0.160)\end{array}$ \\
\hline Conteo de higiene subjetiva & $\begin{array}{l}-0.224^{* * *} \\
(0.083)\end{array}$ & $\begin{array}{l}-0.194^{*} \\
(0.115)\end{array}$ & $\begin{array}{l}-0.190^{*} \\
(0.115)\end{array}$ & $\begin{array}{l}-0.175 \\
(0.120)\end{array}$ \\
\hline Conteo de higiene objetiva & $\begin{array}{l}-0.030 \\
(0.380)\end{array}$ & $\begin{array}{l}-0.241 \\
(0.601)\end{array}$ & $\begin{array}{c}0.155 \\
(0.608)\end{array}$ & $\begin{array}{l}-0.374 \\
(0.781)\end{array}$ \\
\hline Conteo de problemas en los baños & $\begin{array}{l}-0.179 \\
(0.122)\end{array}$ & $\begin{array}{l}-0.247^{*} \\
(0.172)\end{array}$ & $\begin{array}{l}-0.287 \\
(0.211)\end{array}$ & $\begin{array}{l}-0.308^{*} \\
(0.221)\end{array}$ \\
\hline $\begin{array}{l}\text { Conductas no convencionales por } \\
\text { fuera del colegio }\end{array}$ & $\begin{array}{c}0.164 \\
(0.107)\end{array}$ & $\begin{array}{l}0.219^{*} \\
(0.108)\end{array}$ & $\begin{array}{l}0.245^{*} \\
(0.117)\end{array}$ & $\begin{array}{l}1.435^{*} \\
(0.513)\end{array}$ \\
\hline $\begin{array}{l}\text { Conteo de señales de desorden físico } \\
\text { fuera del colegio }\end{array}$ & $\begin{array}{c}0.074 \\
(0.044)\end{array}$ & $\begin{array}{c}0.057 \\
(0.056)\end{array}$ & $\begin{array}{c}0.053 \\
(0.059)\end{array}$ & $\begin{array}{l}-0.001 \\
(0.062)\end{array}$ \\
\hline Ln(Índice de ruralidad) & & $\begin{array}{l}-0.001 \\
(0.036)\end{array}$ & $\begin{array}{c}0.005 \\
(0.046)\end{array}$ & $\begin{array}{c}0.026 \\
(0.026)\end{array}$ \\
\hline Oficial & & $\begin{array}{c}0.266 \\
(0.532)\end{array}$ & $\begin{array}{c}0.238 \\
(0.525)\end{array}$ & $\begin{array}{c}0.290 \\
(0.647)\end{array}$ \\
\hline $\begin{array}{l}\text { Índice de Nivel Socioeconómico (INSE) } \\
\text { del establecimiento }\end{array}$ & & $\begin{array}{l}-0.009 \\
(0.032)\end{array}$ & $\begin{array}{l}-0.012 \\
(0.036)\end{array}$ & $\begin{array}{l}-0.052 \\
(0.047)\end{array}$ \\
\hline $\begin{array}{l}\text { Logaritmo natural del número de } \\
\text { estudiantes en la sede-jornada }\end{array}$ & & $\begin{array}{l}-0.152 \\
(0.178)\end{array}$ & $\begin{array}{l}-0.365^{*} \\
(0.246)\end{array}$ & $\begin{array}{l}0.075 \\
(0.153)\end{array}$ \\
\hline Número de sedes & & $\begin{array}{l}-0.221 \\
(0.228)\end{array}$ & $\begin{array}{l}-0.226 \\
(0.229)\end{array}$ & $\begin{array}{l}-0.109 \\
(0.159)\end{array}$ \\
\hline $\begin{array}{l}\text { Colegio con afiliación religiosa } \\
\text { explícita/observable }\end{array}$ & & $\begin{array}{l}-0.092 \\
(0.308)\end{array}$ & $\begin{array}{l}-0.464^{*} \\
(0.371)\end{array}$ & $\begin{array}{l}0.675 \\
(0.370)\end{array}$ \\
\hline Observador hombre & & $\begin{array}{l}-0.663^{* * *} \\
(0.350)\end{array}$ & $\begin{array}{l}-0.708^{* * *} \\
(0.367)\end{array}$ & $\begin{array}{c}-0.683^{* * *} \\
(0.314)\end{array}$ \\
\hline Señales de desorden*Afiliación religiosa & & & $\begin{array}{c}0.240 \\
(0.148)\end{array}$ & \\
\hline
\end{tabular}




\begin{tabular}{|c|c|c|c|c|}
\hline Variables & Modelo 1 & Modelo 2 & Modelo 3 & Modelo 4 \\
\hline $\begin{array}{l}\text { Señales de desorden*Ln(número } \\
\text { de estudiantes) }\end{array}$ & & & $\begin{array}{c}0.136 \\
(0.104)\end{array}$ & \\
\hline $\begin{array}{l}\text { Señales de desorden*Ln(Índice } \\
\text { de ruralidad) }\end{array}$ & & & $\begin{array}{l}-0.003 \\
(0.015)\end{array}$ & \\
\hline $\begin{array}{l}\text { Conductas no convencionales por fuera } \\
\text { del colegio*Afiliación religiosa }\end{array}$ & & & & $\begin{array}{l}-0.206 \\
(0.204)\end{array}$ \\
\hline $\begin{array}{l}\text { Conductas no convencionales por fuera } \\
\text { del colegio* } \operatorname{Ln} \text { (número de estudiantes) }\end{array}$ & & & & $\begin{array}{l}-0.059 \\
(0.094)\end{array}$ \\
\hline $\begin{array}{l}\text { Conductas no convencionales por fuera } \\
\text { del colegio* } \operatorname{Ln}(\text { Índice de ruralidad) }\end{array}$ & & & & $\begin{array}{l}-0.032^{* *} \\
(0.013)\end{array}$ \\
\hline Observaciones & 148 & 148 & 148 & 148 \\
\hline Pseudo-R2 McFadden & 0.131 & 0.104 & 0.077 & 0.106 \\
\hline AIC & 161.433 & 166.574 & 171.600 & 166.140 \\
\hline BIC & 185.410 & 211.532 & 225.550 & 220.090 \\
\hline
\end{tabular}

Nota: cálculos propios. Errores estándar por clúster de municipio en paréntesis. ${ }^{* * *} \mathrm{p}<0.01,{ }^{* *} \mathrm{p}<0.05,{ }^{*} \mathrm{p}<0.10$. Fuente: elaboración propia.

Las estimaciones de las interacciones sociales no convencionales arrojan resultados similares. Al analizar el modelo sin variables de control (modelo 1) se encuentra que una señal de desorden físico adicional se asocia con un incremento en las interacciones no convencionales y, que esta relación es consistente ante la inclusión de variables de control e interacciones con las conductas no convencionales por fuera de los colegios (exceptuando el modelo 3). En esas estimaciones también se evidencia la relación entre la higiene subjetiva y las interacciones sociales no convencionales al interior de los colegios. El aumento de un espacio bien calificado en términos de higiene está asociado con una disminución de las interacciones sociales no convencionales dentro del colegio en un $17 \%$ (modelo 2).

De igual modo, se encontró que las conductas no convencionales por fuera de los colegios se relacionan positivamente con las interacciones sociales al interior de estos. Esa relación se mantiene estadísticamente significativa al incluir las variables de control y los términos de interacción, exceptuando el modelo 4 . Sobre estas últimas (véase en los anexos figura 5 y 6 para visualizar los resultados de las interacciones), la tabla 3 muestra que, tanto la relación entre las señales de desorden físico como la de las conductas no convencionales por fuera de los colegios con las interacciones sociales no convencionales dentro de estos, es moderada por la afiliación religiosa del colegio. En el caso de la interacción con desorden físico, se evidenció que en los colegios 
con una afiliación religiosa explícita y con mayores señales de este tipo de desorden, hay más interacciones sociales no convencionales. Lo contrario sucede en colegios que cuentan con afiliación religiosa pero que a su vez tienen mayores señales de conductas no convencionales por fuera del colegio, en donde se encuentra una relación negativa. Este resultado podría estar reflejando el papel que juega la religión como moderador de las conductas de los estudiantes solo cuando el ambiente externo del colegio se caracteriza por mayor desorden social, algo que no sucede cuando la afiliación religiosa no se emplea como variable moderadora.

En otras palabras, en instituciones con indicios explícitos de afiliación religiosa, hay más interacciones sociales no convencionales cuando se analiza de manera conjunta con el desorden físico, pero menos, cuando a su vez hay más conductas no convencionales su exterior.

Tabla 3. Regresiones Poisson-Interacciones sociales no convencionales

\begin{tabular}{|c|c|c|c|c|}
\hline Variables & Modelo 1 & Modelo 2 & Modelo 3 & Modelo 4 \\
\hline $\begin{array}{l}\text { Conteo de señales de desorden físico } \\
\text { dentro del colegio }\end{array}$ & $\begin{array}{l}0.392^{* *} \\
(0.160)\end{array}$ & $\begin{array}{l}0.330^{*} \\
(0.155)\end{array}$ & $\begin{array}{l}-0.095 \\
(0.838)\end{array}$ & $\begin{array}{l}0.350^{* *} \\
(0.133)\end{array}$ \\
\hline Conteo de ambiente de inseguridad & $\begin{array}{l}-0.094 \\
(0.150)\end{array}$ & $\begin{array}{l}-0.090 \\
(0.159)\end{array}$ & $\begin{array}{l}-0.084 \\
(0.146)\end{array}$ & $\begin{array}{l}-0.062 \\
(0.151)\end{array}$ \\
\hline Conteo de higiene subjetiva & $\begin{array}{l}-0.109 \\
(0.087)\end{array}$ & $\begin{array}{l}-0.173^{*} \\
(0.099)\end{array}$ & $\begin{array}{l}-0.162^{*} \\
(0.100)\end{array}$ & $\begin{array}{l}-0.151^{*} \\
(0.096)\end{array}$ \\
\hline Conteo de higiene objetiva & $\begin{array}{c}0.336 \\
(0.307)\end{array}$ & $\begin{array}{c}0.642 \\
(0.452)\end{array}$ & $\begin{array}{l}1.853^{* *} \\
(0.504)\end{array}$ & $\begin{array}{c}0.154 \\
(0.346)\end{array}$ \\
\hline Conteo de problemas en los baños & $\begin{array}{c}0.088 \\
(0.126)\end{array}$ & $\begin{array}{c}0.135 \\
(0.147)\end{array}$ & $\begin{array}{c}0.156 \\
(0.204)\end{array}$ & $\begin{array}{c}0.165 \\
(0.140)\end{array}$ \\
\hline $\begin{array}{l}\text { Conductas no convencionales por } \\
\text { fuera del colegio }\end{array}$ & $\begin{array}{l}0.214^{* * *} \\
(0.072)\end{array}$ & $\begin{array}{l}0.186^{*} \\
(0.092)\end{array}$ & $\begin{array}{l}0.203^{*} \\
(0.095)\end{array}$ & $\begin{array}{l}-0.178 \\
(0.744)\end{array}$ \\
\hline $\begin{array}{l}\text { Conteo de señales de desorden físico } \\
\text { fuera del colegio }\end{array}$ & $\begin{array}{c}0.049 \\
(0.042)\end{array}$ & $\begin{array}{c}0.070 \\
(0.055)\end{array}$ & $\begin{array}{c}0.072 \\
(0.057)\end{array}$ & $\begin{array}{c}0.066 \\
(0.057)\end{array}$ \\
\hline Ln(Índice de ruralidad) & & $\begin{array}{l}-0.030 \\
(0.028)\end{array}$ & $\begin{array}{c}0.032 \\
(0.035)\end{array}$ & $\begin{array}{l}-0.059 \\
(0.045)\end{array}$ \\
\hline Oficial & & $\begin{array}{l}-0.537 \\
(0.585)\end{array}$ & $\begin{array}{l}-0.619^{* *} \\
(0.418)\end{array}$ & $\begin{array}{l}-0.627 \\
(0.646)\end{array}$ \\
\hline $\begin{array}{l}\text { Índice de Nivel Socioeconómico (INSE) } \\
\text { del establecimiento }\end{array}$ & & $\begin{array}{l}-0.017 \\
(0.044)\end{array}$ & $\begin{array}{l}-0.037 \\
(0.052)\end{array}$ & $\begin{array}{l}-0.017 \\
(0.039)\end{array}$ \\
\hline $\begin{array}{l}\text { Logaritmo natural del número } \\
\text { de estudiantes en la sede-jornada }\end{array}$ & & $\begin{array}{c}0.245 \\
(0.239)\end{array}$ & $\begin{array}{c}0.297 \\
(0.372)\end{array}$ & $\begin{array}{c}0.092 \\
(0.311)\end{array}$ \\
\hline Número de sedes & & $\begin{array}{c}0.259 \\
(0.141)\end{array}$ & $\begin{array}{c}0.334^{* *} \\
(0.144)\end{array}$ & $\begin{array}{c}0.359^{* *} \\
(0.137)\end{array}$ \\
\hline
\end{tabular}




\begin{tabular}{|c|c|c|c|c|}
\hline Variables & Modelo 1 & Modelo 2 & Modelo 3 & Modelo 4 \\
\hline $\begin{array}{l}\text { Colegio con afiliación religiosa } \\
\text { explícita/observable }\end{array}$ & & $\begin{array}{c}0.214 \\
(0.285)\end{array}$ & $\begin{array}{l}-0.782^{*} \\
(0.863)\end{array}$ & $\begin{array}{c}1.141 \\
(0.484)\end{array}$ \\
\hline Observador hombre & & $\begin{array}{c}1.684^{* * *} \\
(0.264)\end{array}$ & $\begin{array}{c}1.441^{* * *} \\
(0.263)\end{array}$ & $\begin{array}{c}2.024^{* * *} \\
(0.272)\end{array}$ \\
\hline $\begin{array}{l}\text { Señales de desorden*Afiliación } \\
\text { religiosa }\end{array}$ & & & $\begin{array}{l}0.868^{* *} \\
(0.272)\end{array}$ & \\
\hline $\begin{array}{l}\text { Señales de desorden*Ln(número } \\
\text { de estudiantes) }\end{array}$ & & & $\begin{array}{c}0.033 \\
(0.107)\end{array}$ & \\
\hline $\begin{array}{l}\text { Señales de desorden*Ln(Índice } \\
\text { de ruralidad) }\end{array}$ & & & $\begin{array}{l}-0.024 \\
(0.016)\end{array}$ & \\
\hline $\begin{array}{l}\text { Conductas no convencionales por } \\
\text { fuera del colegio*Afiliación religiosa }\end{array}$ & & & & $\begin{array}{c}-0.248^{* *} \\
(0.142)\end{array}$ \\
\hline $\begin{array}{l}\text { Conductas no convencionales por } \\
\text { fuera del colegio* } \operatorname{Ln} \text { (número de } \\
\text { estudiantes) }\end{array}$ & & & & $\begin{array}{c}0.071 \\
(0.112)\end{array}$ \\
\hline $\begin{array}{l}\text { Conductas no convencionales por fue- } \\
\text { ra del colegio* } \operatorname{Ln}(\text { Índice de ruralidad) }\end{array}$ & & & & $\begin{array}{c}0.013 \\
(0.010)\end{array}$ \\
\hline Observaciones & 148 & 148 & 148 & 148 \\
\hline Pseudo-R2 McFadden & 0.094 & 0.086 & 0.094 & 0.083 \\
\hline AIC & 215.538 & 217.509 & 215.563 & 218.161 \\
\hline BIC & 239.516 & 262.467 & 269.512 & 272.111 \\
\hline
\end{tabular}

Por último, aunque no hace parte de las variables de interés, es importante resaltar la relación entre ser observador hombre y las medidas de desorden social dentro de los colegios, ya que esta resultó ser estadísticamente significativa en todos los modelos en los que se incluyó como control. El hecho de que el observador sea hombre está asociado con menores conductas no convencionales, pero con mayores interacciones sociales no convencionales al interior de los colegios. Lo anterior indica que las características de los observadores pueden ser una fuente de sesgo que deben ser tenidas en cuenta en las estimaciones, tal y como se hace con la inclusión del sexo del observador como variable de control en los modelos.

Con el fin de determinar cuál de los modelos estimados se ajusta mejor a los datos, se emplearon varias medidas de ajuste y criterios de información. Por un lado, al ver los valores del pseudo-R cuadrado ajustado de McFadden, se ve que el modelo con mayor ajuste es aquel que no incluye los controles de las sedes-jornada ni las variables de interacción para ambas variables dependientes. Sin embargo, estos coeficientes también indican que los modelos 
no cuentan con un buen ajuste (McFadden, 1977). Teniendo en cuenta esto, y que los coeficientes que emplea la lógica de R-cuadrado no son la mejor aproximación a los ejercicios realizados con el método de máxima verosimilitud, se decidió observar criterios de información. Específicamente, el Criterio de Información de Akaike (AIC, por su sigla en inglés) y el Criterio de Información Bayesiano (BIC, por su sigla en inglés) con lo cual se encontraron resultados similares soportando la idea de que el mejor modelo para emplear es aquel en el cual no se incluyen las variables de control.

Asimismo, se quiso analizar si los resultados asociados con la variable de conductas no convencionales eran sensibles al peso que puede tener el observar estudiantes con armas dentro de los colegios, ya que esto puede estar asociado a conductas violentas e ilegales que difieren sustancialmente de las demás variables con las que se construyó el indicador. Para esto, se realizó un análisis de sensibilidad en el que se aumentó el peso que la variable de estudiantes con armas tiene sobre el conteo de conductas no convencionales, peso que va desde 1 (modelo original) hasta 5, manteniendo el peso de las demás variables explicativas constante (peso 1). Los resultados de estos análisis muestran que en el modelo sin variables de control — según lo discutido, el mejor modelo- las señales de desorden dentro del colegio y la higiene subjetiva continúan teniendo una relación significativa y, en la dirección original cuando a la variable de estudiantes con armas al interior de las instituciones educativas se le da un peso de hasta 4 en la construcción del conteo de conductas no convencionales, aunque el nivel de significancia se va reduciendo a medida que aumenta el peso (véase en los anexos tabla 6). Asimismo, se observó que al aumentar el peso de esta variable sobre las conductas no convencionales, otras señales de desorden empezaron a ser significativas. Este es el caso de los problemas en los baños, conductas no convencionales por fuera de los colegios y las señales de desorden físico por fuera de los colegios, resultados que no eran significativos en las estimaciones en las que se asumió el mismo peso para todas las variables con las que se construyó el conteo de conductas no convencionales.

Finalmente, se realizó un ejercicio de validez en el que se contrastaron los valores observados de las variables dependientes con sus respectivas estimaciones. Los resultados de este ejercicio, que se presentan en la figura 2, indican que los modelos empleados son adecuados para analizar las relaciones de interés. Esto en la medida en que las diferencias entre los valores estimados y los valores observados de las variables de resultado son como máximo 0.5. Es decir que, en promedio, las estimaciones sí están reflejando los valores efectivamente observados. 

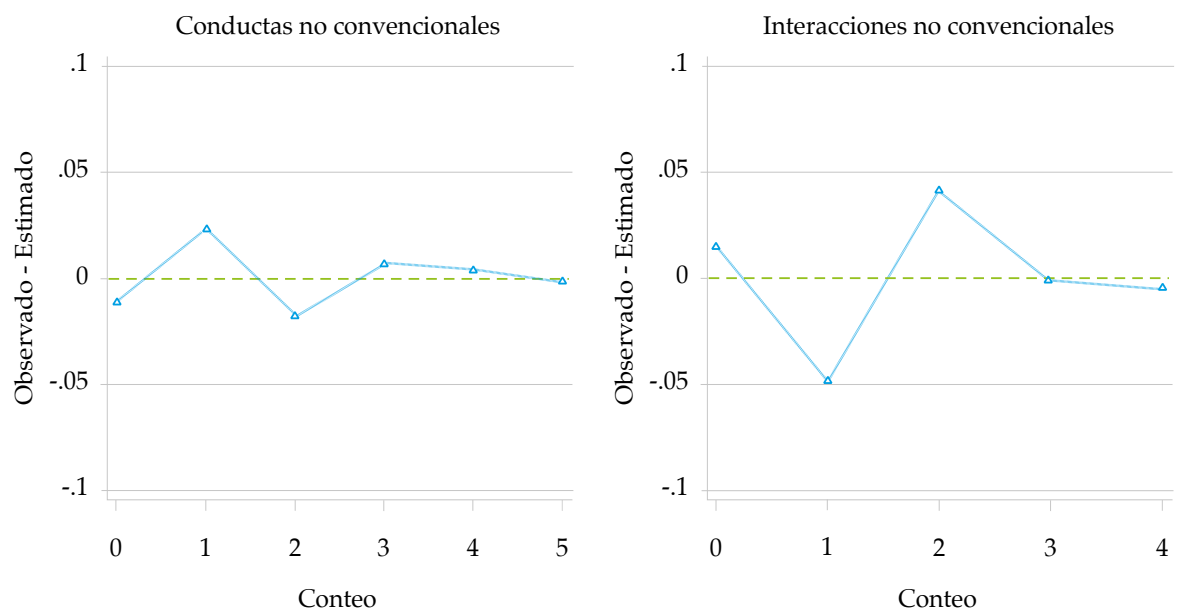

Figura 2. Resultados estimados vs. Observados Fuente: elaboración propia.

\section{Discusión}

Este trabajo de investigación buscaba analizar la relación entre las características físicas y sociales de los entornos escolares. Así, el conjunto de colegios observados se caracterizó por tener más interacciones sociales no convencionales que conductas no convencionales, relativamente tienen pocas señales de desorden físico en su interior, poco ambiente de inseguridad y pocas señales de desorden físico en su entorno. Asimismo, en promedio, los colegios analizados tienen buena higiene, medida tanto de forma objetiva como subjetiva y, en su exterior se presentan conductas no convencionales de manera considerable. Con respecto a la relación entre estas variables, se ve una asociación estadísticamente significativa entre el desorden físico en los establecimientos educativos y los comportamientos e interacciones no convencionales de los estudiantes.

$\mathrm{Al}$ encontrar que la relación entre las señales de desorden físico dentro de los colegios y las señales de desorden social dentro de estos es positiva, es notable que, para el conjunto de colegios observados, se cumple lo establecido por la teoría de las ventanas rotas aplicado al contexto educativo. En este sentido, este estudio aporta a la literatura existente ya que se evidencia que las señales de desorden físico dentro de las instituciones educativas pueden estar promoviendo un ciclo de trasgresiones al orden convencional en el ambiente educativo. De igual manera, y tomando en cuenta lo mencionado en el marco teórico, se evidencia que este tipo de desorden también puede estar siendo interpretado por los estudiantes como una señal de control social 
débil, lo cual incrementa sus conductas e interacciones no convencionales al percibir una menor probabilidad de ser sancionados.

Otro resultado que refuerza la hipótesis anterior, es el relacionado con la higiene subjetiva. Los análisis muestran que la variable está relacionada negativamente con las conductas e interacciones sociales no convencionales. De manera que, se puede decir que un ambiente que es valorado por los individuos como higiénico puede ser interpretado como una señal de que ese espacio es cuidado y bien mantenido y, por ende, es una muestra de mayor control social. Así, al haber un control social fuerte, como lo dice la teoría de las ventanas rotas, los estudiantes perciben una mayor probabilidad de ser sancionados.

De estos resultados, también es de destacar la relación existente entre el ambiente externo de los colegios y el desorden social en su interior. Específicamente, hay evidencia de que las conductas no convencionales por fuera del colegio se relacionan positivamente con las conductas e interacciones no convencionales dentro de las instituciones educativas. En este sentido, es posible pensar que el ambiente social al exterior de los colegios puede estar influyendo en los comportamientos de los estudiantes en su interior. Aunque no se ahondó en los mecanismos que subyacen esta relación, los resultados de las regresiones que incluyen términos de interacción indican que existen factores como la afiliación religiosa y el grado de ruralidad, que pueden estar moderando la relación observada.

Con respecto a la metodología, es de reconocer que, puesto que no se empleó ningún método para reducir el sesgo generado por el incumplimiento del supuesto de independencia condicional del error, los estimados presentados no pueden tomarse como relaciones causales. Más aún, dada la manera en que se seleccionaron las sedes-jornadas a visitar, no es posible afirmar que los resultados sean extrapolables al conjunto global de los colegios de Colombia o, que se cumple a cabalidad el supuesto de estabilidad del valor de la unidad de tratamiento ${ }^{15}$ (sUTva, por sus siglas en inglés).

Adicionalmente, vale la pena resaltar que la operacionalización de las variables es limitada debido a que el enfoque son los comportamientos de los estudiantes, ignorando los comportamientos de actores que también pueden ser relevantes, como los docentes. Se considera que, aunque esta limitación puede ser abordada en estudios etnográficos, es importante tener presente el número limitado de observaciones, y por tanto, los mayores problemas de validez externa que estos suelen tener.

15 Dada la cercanía de los colegios analizados, resulta probable que el desorden físico que rodea a una sede-jornada también tenga un efecto sobre otras sedes-jornada de la zona. 
Así mismo vale la pena resaltar otras dos limitaciones de este estudio. Primero, la presentación de una prueba de estado puede afectar considerablemente el comportamiento de los estudiantes, el personal administrativo y el personal docente, en lo referente al orden del colegio - e.g. los estudiantes podrían evitar arrojar basuras a su alrededor o los profesores podrían estar más pendientes del comportamiento de sus estudiantes en comparación a un día normal- Segundo, existe un problema de simultaneidad que se ha reconocido en esta literatura entre el desorden físico y el desorden social. Esto es, así como mayores señales de desorden físico como grafitis o basuras pueden promover mayor desorden social, mayores señales de desorden social como abuso verbal o peleas pueden propiciar un mayor desorden físico.

A pesar de estas limitaciones, los resultados fueron consecuentes con lo encontrado por Plank et al. (2009), quienes empleando modelos de ecuaciones estructurales, también encontraron que el desorden social es un factor importante para explicar, entre otros, el comportamiento de los estudiantes. De esta manera, los resultados sirven como motivación para realizar este tipo de análisis avanzando con muestras de representatividad a nivel nacional o con el uso de metodologías que no se limiten a datos de corte transversal, esto con el fin de evaluar la consistencia de las relaciones acá presentadas. Por el momento, los resultados sirven como evidencia de que las instituciones educativas que buscan formar a sus estudiantes, no solo académicamente sino también como ciudadanos, deben prestar atención a las señales que pueden interferir con este objetivo. Más aún, que el cumplimento de este objetivo no es responsabilidad única de los estudiantes y los maestros, sino que también, se debe en parte al mantenimiento y cuidado de las instalaciones de los colegios.

\section{Referencias}

Andrade Álvarez, M. (2011). Religión, política y educación en Colombia. La presencia religiosa extranjera en la consolidación del régimen conservador durante la Regeneración. Revista de Historia Regional y Local, 3(6), 154-171. https://www.redalyc.org/pdf/3458/345832079007.pdf

Berger, J., \& Hevenstone, D. (2016). Norm enforcement in the city revisited: An international field experiment of altruistic punishment, norm maintenance, and broken windows. Rationality and Society, 28(3), 299-319. https://doi.org/10.1177/1043463116634035

Bradshaw, C. P., Milam, A. J., Furr-Holden, C. D. M., \& Johnson, S. L. (2015). The School Assessment for Environmental Typology (SAFETY): An 
observational measure of the school environment. American Journal of Community Psychology, 56(3-4), 280-292. https://doi.org/10.1007/s10464015-9743-x

Branas, C. C., Kondo, M. C., Murphy, S. M., South, E. C., Polsky, D., \& MacDonald, J. M. (2016). Urban blight remediation as a cost-beneficial solution to firearm violence. American Journal of Public Health, 106(12), 2158-2164. https://doi.org/10.2105/AJPH.2016.303434

Branas, C. C., South, E., Kondo, M. C., Hohl, B. C., Bourgois, P., Wiebe, D. J., \& MacDonald, J. M. (2018). Citywide cluster randomized trial to restore blighted vacant land and its effects on violence, crime, and fear. Proceedings of the National Academy of Sciences, 115(12), 2946-2951. https://doi. org/10.1073/pnas.1718503115

Brown, B. B., Perkins, D. D., \& Brown, G. (2004). Incivilities, place attachment and crime: block and individual effects. Journal of Environmental Psychology, 24(3), 359-371. https://doi.org/10.1016/j.jenvp.2004.01.001

Cameron, A. C., \& Trivedi, P. K. (1998). Regression analysis of count data. Cambridge University Press.

Cerdá, M., Morenoff, J. D., Hansen, B. B., Tessari Hicks, K. J., Duque, L. F., Restrepo, A., \& Diez-Roux, A. V. (2012). Reducing violence by transforming neighborhoods: a natural experiment in Medellín, Colombia. American Journal of Epidemiology, 175(10), 1045-1053. https://doi.org/10.1093/aje/ kwr428

Chappell, A. T., Monk-Turner, E., \& Payne, B. K. (2011). Broken windows or window breakers: The influence of physical and social disorder on quality of life. Justice Quarterly, 28(3), 522-540. https://doi.org/10.1080/ 07418825.2010.526129

Gau, J. M., \& Pratt, T. C. (2008). Broken windows or window dressing? Citizens'(in) ability to tell the difference between disorder and crime. Criminology \& Public Policy, 7(2), 163-194. https://doi.org/10.1111/j.17459133.2008.00500.x

Janowitz, M. (1975). Sociological theory and social control. American Journal of Sociology, 81(1), 82-108. https://doi.org/10.1086/226035

Keuschnigg, M., \& Wolbring, T. (2015). Disorder, social capital, and norm violation: Three field experiments on the broken windows thesis. Rationality and Society, 27(1), 96-126. https://doi.org/10.1177/1043463114561749

Kondo, M. C., Andreyeva, E., South, E. C., MacDonald, J. M., \& Branas, C. C. (2018). Neighborhood interventions to reduce violence. Annual Review of Public Health, 39, 253-271. https://doi.org/10.1146/annurevpublhealth-040617-014600 
Kubrin, C. E. (2008). Making order of disorder: A call for conceptual clarity. Criminology and Public Policy, 7(2), 203-214. https://heinonline.org/HOL/ LandingPage?handle=hein.journals/crpp7\&div $=22 \& i d=\&$ page $=$

Lanfear, C. C., Matsueda, R. L., \& Beach, L. R. (2020). Broken windows, informal social control, and crime: assessing causality in empirical studies. Annual Review of Criminology, 3(1), 97-120. https://doi.org/10.1146/ annurev-criminol-011419-041541

Lilly, J. R., Cullen, F. T., \& Ball, R. A. (2002). Criminological Theory: Context and Consequences (3ra Ed). Sage Publications.

Logan, T. K., \& Walker, R. (2017). The gender safety gap: examining the impact of victimization history, perceived risk, and personal control. Journal of Interpersonal Violence. https://doi.org/10.1177/0886260517729405

Messner, S. F. (1988). Merton's 'social structure and anomie': the road not taken. Deviant Behavior, 9(1), 33-53. https://doi.org/10.1080/01639625.1 988.9967766

Mijanovich, T., \& Weitzman, B. C. (2003). Which "broken windows" matter? School, neighborhood, and family characteristics associated with youths' feelings of unsafety. Journal of Urban Health, 80(3), 400-415. https://doi. org/10.1093/jurban/jtg045

Milam, A. J., Furr-Holden, c. D. M., \& Leaf, P. J. (2010). Perceived school and neighborhood safety, neighborhood violence and academic achievement in urban school children. The Urban Review, 42(5), 458-467. https://doi. org/10.1007/s11256-010-0165-7

Plank, S. B., Bradshaw, C. P., \& Young, H. (2009). An application of "broken windows" and related theories to the study of disorder, fear, and collective efficacy in schools. American Journal of Education, 115(2), 227-247. https://doi.org/10.1086/595669

Pratt, T. C., \& Cullen, F. T. (2005). Assessing macro-level predictors and theories of crime: a meta-analysis. Crime and Justice, 32, 373-450. https://doi. org $/ 10.1086 / 655357$

Ross, C. E., \& Mirowsky, J. (1999). Disorder and decay: The concept and measurement of perceived neighborhood disorder. Urban Affairs Review, 34(3), 412-432. https://doi.org/10.1177/107808749903400304

Sampson, R. J., \& Groves, W. B. (1989). Community structure and crime: Testing social-disorganization theory. American Journal of Sociology, 94(4), 774-802. https://doi.org/10.1086/229068

Sampson, R. J., \& Raudenbush, S. W. (1999). Systematic social observation of public spaces: A new look at disorder in urban neighborhoods. American Journal of Sociology, 105(3), 603-651. https://doi.org/10.1086/210356 
Sampson, R. J., Raudenbush, S. W., \& Earls, F. (1997). Neighborhoods and violent crime: A multilevel study of collective efficacy. Science, 277(5328), 918-924. https://doi.org/10.1126/science.277.5328.918

Shaw, C. R., \& McKay, H. D. (1931). Report on the Causes of Crime (Vol. II). Government Publishing Office.

Shaw, C. R., \& McKay, H. D. (1942). Juvenile delinquency and urban areas. The University of Chicago Press.

Skogan, W. G. (1990). Disorder and decline: crime and the spiral decay in American cities. Free Press.

Stebbins, R. A. (2011). The semiotic self and serious leisure. The American Sociologist, 42(2-3), 238-248. https://doi.org/10.1007/s12108-011-9126-1

Uline, C., \& Tschannen-Moran, M. (2008). The walls speak: The interplay of quality facilities, school climate, and student achievement. Journal of Educational Administration, 46(1), 55-73. https://doi.org/10.1108/09578230810849817

Villarreal, A., \& Silva, B. F. (2006). Social cohesion, criminal victimization and perceived risk of crime in Brazilian neighborhoods. Social Forces, 84(3), 1725-1753. https://doi.org/10.1353/sof.2006.0073

Weber, M. (1964). Economía y sociedad. Fondo de Cultura Económica.

Wilcox, P., Augustine, M. C., \& Clayton, R. R. (2006). Physical environment and crime and misconduct in Kentucky schools. Journal of Primary Prevention, 27(3), 292-313. https://doi.org/10.1007/s10935-006-0034-z

Wilson, J. Q., \& Kelling, G. L. (1982). Broken windows. Atlantic monthly, 249(3), 29-38. http://illinois-online.org/krassa/ps410/Readings/Wilson\%20 and\%20Kelling\%20Broken\%20Windows.pdf

\section{Anexos}

Tabla 4. Características del entorno interno del colegio

\begin{tabular}{lll}
\hline Aspecto & Variables de conteo & \multicolumn{1}{c}{ Variables dicótomas (ítems) } \\
\hline & & $\begin{array}{l}\text { Estudiantes con piercings, pelo de colores, uñas pintadas, } \\
\text { tatuajes o no uniformados }\end{array}$ \\
& $\begin{array}{l}\text { Conductas no } \\
\text { convencionales } \\
\text { orden } \\
\text { social }\end{array}$ & Estudiantes embarazadas \\
& & $\begin{array}{l}\text { Estudiantes con armas (e.g. cuchillos, elementos corto-pun- } \\
\text { zantes, armas de fuego) }\end{array}$ \\
& & Estudiantes fumando \\
& $\begin{array}{l}\text { Interacciones no } \\
\text { convencionales }\end{array}$ & Estudiantes peleando o discutiendo acaloradamente \\
& & Demostraciones de afecto excesivas
\end{tabular}




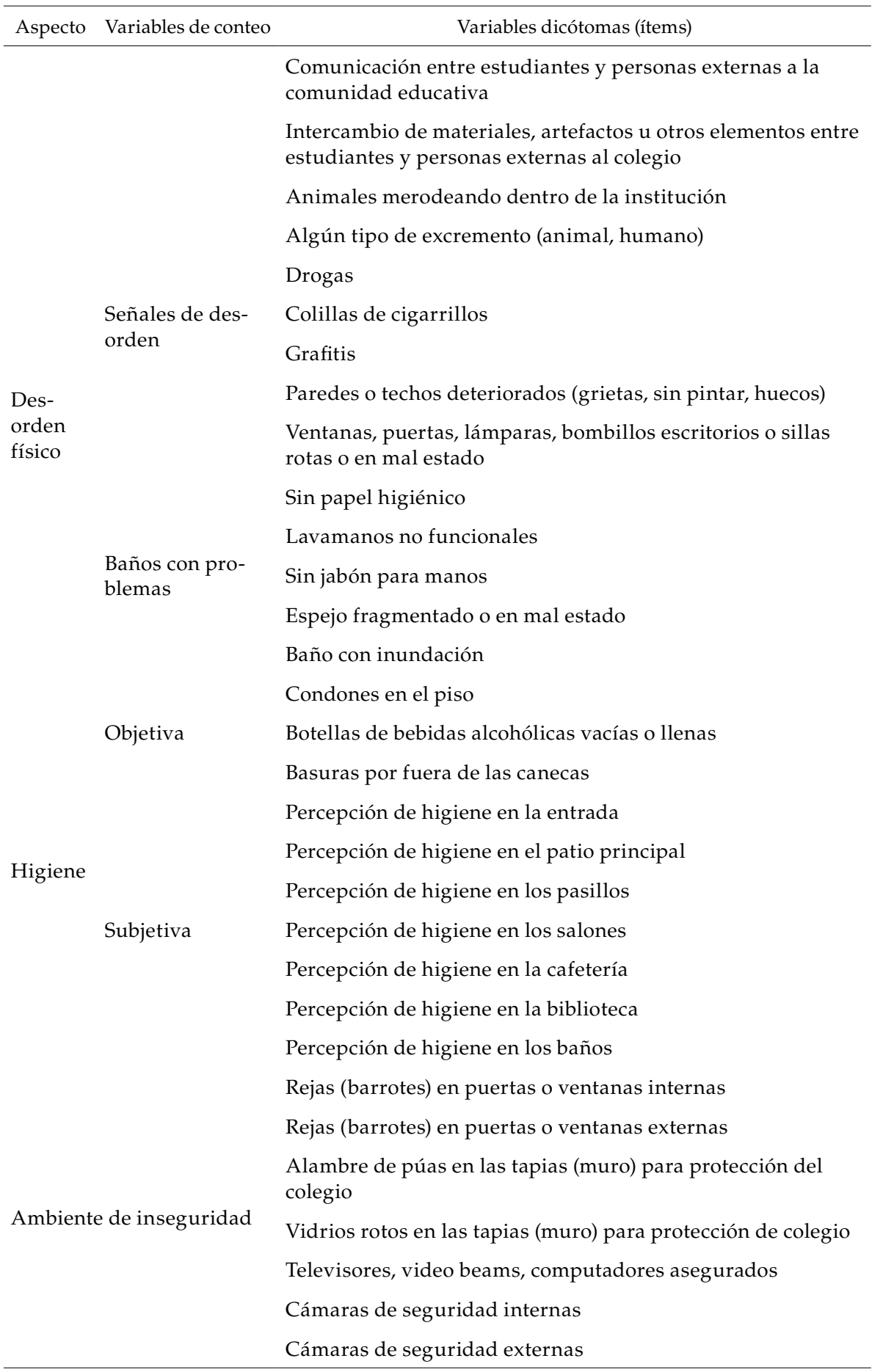

Fuente: elaboración propia. 
Tabla 5. Características del entorno externo del colegio

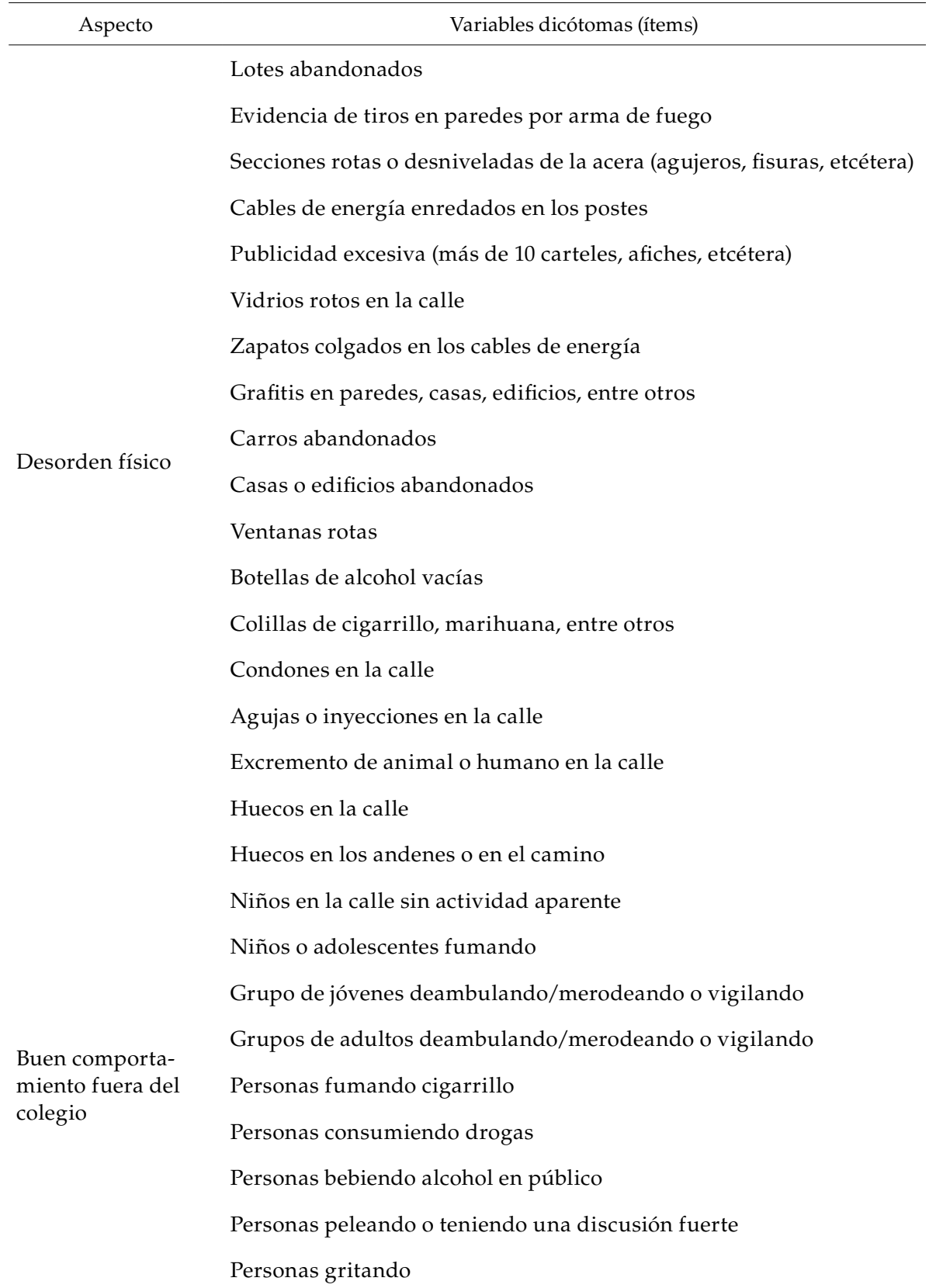




\begin{tabular}{ll}
\hline \multicolumn{1}{c}{ Aspecto } & \multicolumn{1}{c}{ Variables dicótomas (ítems) } \\
\hline & $\begin{array}{l}\text { Instituciones estatales (estación de policía, comisaría, etcétera) } \\
\text { Presencia de policía(s) o ejército }\end{array}$ \\
$\begin{array}{l}\text { Regulación del } \\
\text { espacio }\end{array}$ & $\begin{array}{l}\text { Presencia de guardas de seguridad (formales o informales) } \\
\text { Iglesias } \\
\end{array}$ \\
& Guarderías, jardines \\
& Habitantes de calle \\
& Vendedores ambulantes \\
Ambiente social & Personas vendiendo cigarrillos en la calle \\
& Personas vendiendo drogas \\
\hline
\end{tabular}

Fuente: elaboración propia.

Tabla 6. Análisis de sensibilidad: conductas no convencionales peso estudiantes con armas

\begin{tabular}{llllll}
\hline \multicolumn{1}{c}{ Variable } & Peso=1 & Peso $=2$ & Peso $=3$ & Peso $=4$ & Peso =5 \\
\hline Conteo de señales de desorden físico & $0.26^{* *}$ & $0.23^{* *}$ & $0.21^{*}$ & $0.19^{*}$ & 0.17 \\
dentro del colegio & $(0.11)$ & $(0.11)$ & $(0.10)$ & $(0.10)$ & $(0.10)$ \\
& 0.03 & 0.02 & 0.00 & -0.01 & -0.02 \\
Conteo de ambiente de inseguridad & $(0.11)$ & $(0.11)$ & $(0.11)$ & $(0.11)$ & $(0.12)$ \\
& $-0.22^{* * *}$ & $-0.24^{* * *}$ & $-0.25^{* * *}$ & $-0.26^{* * *}$ & $-0.27^{* * *}$ \\
Conteo de higiene subjetiva & $(0.08)$ & $(0.08)$ & $(0.08)$ & $(0.08)$ & $(0.08)$ \\
& -0.03 & -0.04 & -0.05 & -0.05 & -0.05 \\
Conteo de higiene objetiva & $(0.38)$ & $(0.34)$ & $(0.32)$ & $(0.31)$ & $(0.30)$ \\
& & & & & \\
Conteo de problemas en los baños & -0.18 & $-0.18^{*}$ & $-0.17^{*}$ & $-0.17^{*}$ & $-0.17^{*}$ \\
& $(0.12)$ & $(0.12)$ & $(0.11)$ & $(0.11)$ & $(0.11)$ \\
Conductas no convencionales por & 0.16 & $0.20^{*}$ & $0.23^{* *}$ & $0.26^{* *}$ & $0.29^{* *}$ \\
fuera del colegio & $(0.11)$ & $(0.10)$ & $(0.10)$ & $(0.10)$ & $(0.10)$ \\
Conteo de señales de desorden físico & 0.07 & $0.08^{*}$ & $0.09^{* *}$ & $0.09^{* *}$ & $0.10^{* *}$ \\
fuera del colegio & $(0.04)$ & $(0.04)$ & $(0.04)$ & $(0.04)$ & $(0.04)$ \\
\hline
\end{tabular}

Nota: cálculos propios. Errores estándar por clúster de municipio en paréntesis. ${ }^{* *} \mathrm{p}<0.01,{ }^{* *} \mathrm{p}<0.05,{ }^{*} \mathrm{p}<0.10$. Fuente: elaboración propia. 


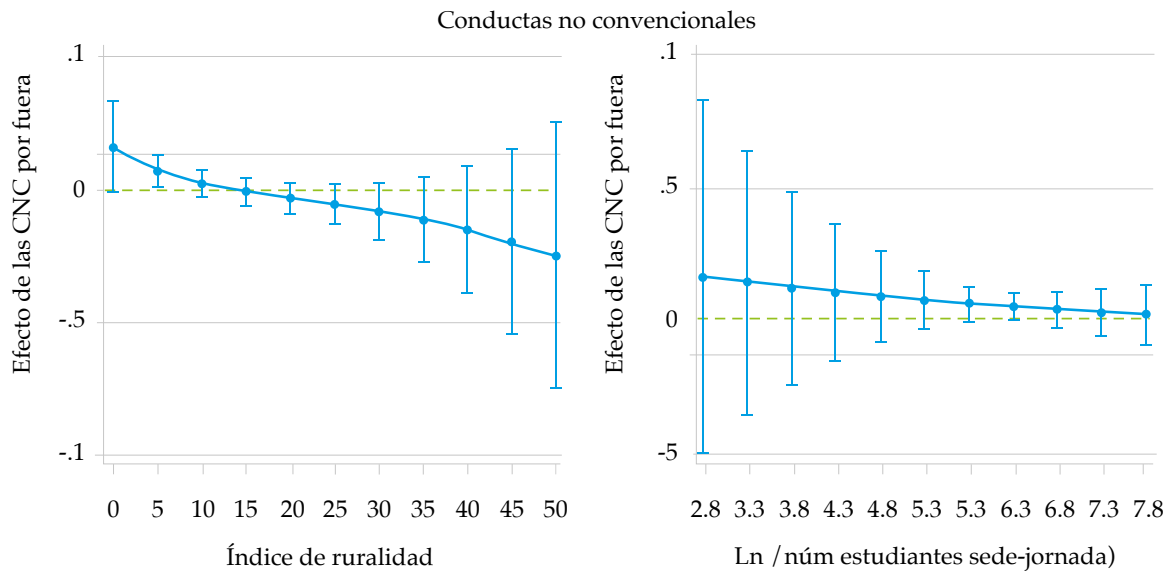

Figura 3. Interacciones de conductas no convencionales por fuera de los colegios: conductas no convencionales dentro de los colegios

Fuente: elaboración propia.

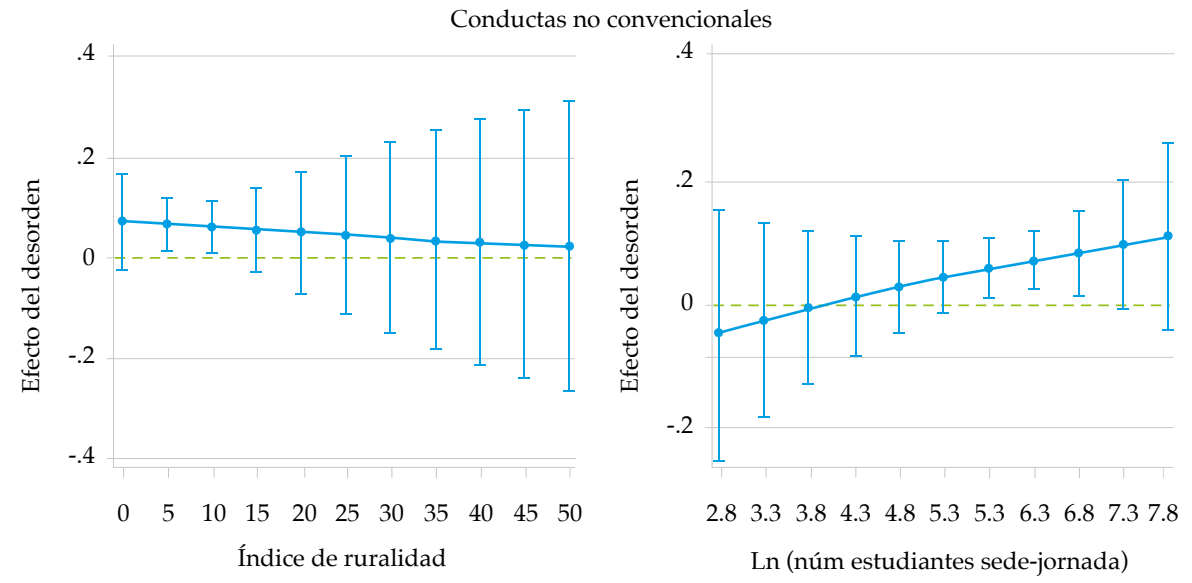

Figura 4. Interacciones de señales de desorden físico por fuera de los colegios: conductas no convencionales dentro de los colegios

Fuente: elaboración propia. 


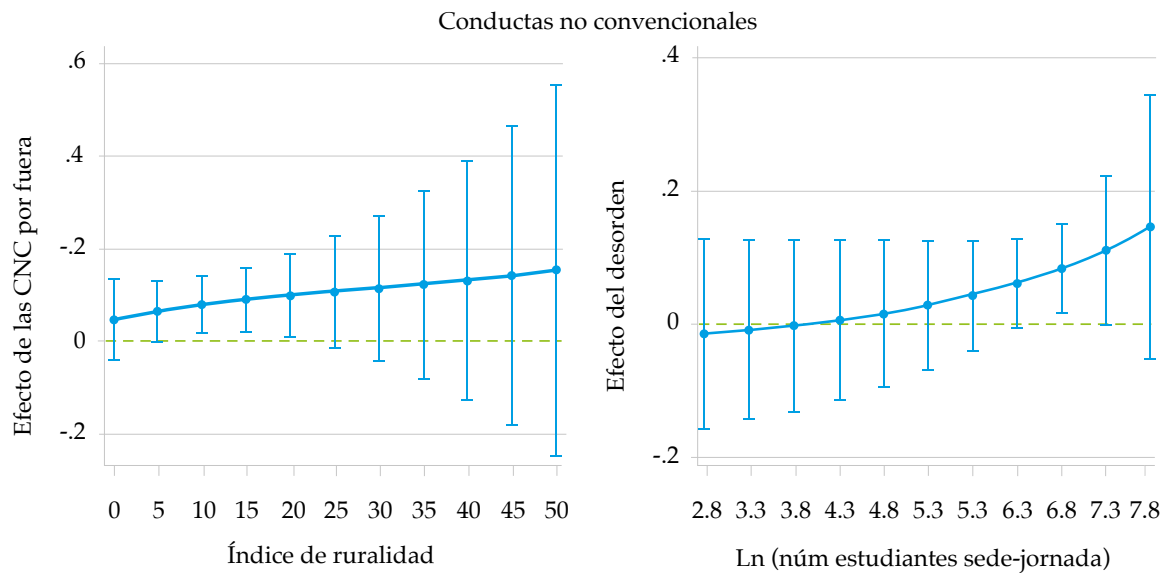

Figura 5. Interacciones de conductas no convencionales por fuera de los colegios: interacciones sociales no convencionales dentro de los colegios Fuente: elaboración propia.

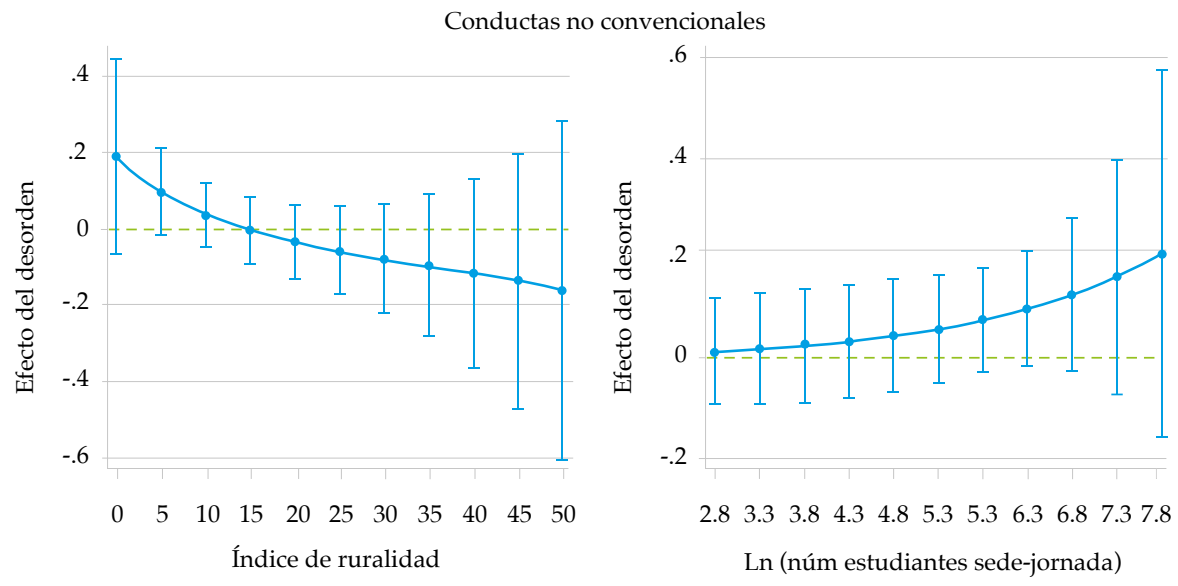

Figura 6. Interacciones de señales de desorden físico por fuera de los colegios: interacciones sociales no convencionales dentro de los colegios Fuente: elaboración propia. 